\title{
Effects of Intragastric Sodium Bicarbonate in Infants with Respiratory Distress
}

\author{
MARGARET E. R. STONEMAN and ROSALIND M. OWENS \\ From the Paediatric Research Unit and Special Care Baby Unit, Exeter City Hospital
}

Since Usher reported a significant fall in mortality in infants with respiratory distress who were treated with intravenous glucose and sodium bicarbonate (Usher, 1959, 1960, 1961, 1963), this form of treatment has been widely adopted (Warley and Gairdner, 1962; Hutchison et al., 1962, 1964; Gairdner, 1965). Intragastric glucose and sodium bicarbonate have also been used (Abraham and Brown, 1967).

Feeding of healthy premature babies, beginning at the age of 3 or 4 hours, was being carried out with success in the Special Care Baby Unit of Exeter City Hospital when facilities for biochemical investigation of cases of respiratory distress became available in 1963. A decision was, therefore, made to undertake a trial of treatment of cases of respiratory distress by means of intragastric sodium bicarbonate. Our experience has shown that the value of this treatment is limited, and that in some cases it may possibly be harmful. This form of treatment has therefore been discontinued in all except a minority of cases. The results of our observations on babies treated with and without intragastric sodium bicarbonate are reported in the present paper. The indications and contraindications for such treatment are discussed.

\section{Material and Methods}

All the patients were admitted to the Special Care Baby Unit of Exeter City Hospital between August 1963 and July 1967. A diagnosis of respiratory distress was made if two or more of the following criteria were present after the age of 1 hour: (1) grunting respiration; (2) sternal or intercostal recession; (3) cyanosis; (4) respiratory rate of 65 or more, on more than one recording at 3-hourly intervals.

Of 390 babies who fulfilled these criteria, 24, whose respiratory distress was due to miscellaneous causes such as diaphragmatic hernia, pneumothorax, and massive meconium aspiration have been excluded, as have 18 babies who died from causes other than respiratory distress. It was also necessary to exclude the effects of

Received October 16, 1967. intermittent positive pressure ventilation (IPPV) on biochemical findings and on mortality. For this reason 16 further cases have been excluded: 7 survivors, because it was considered that their survival would have been extremely unlikely without IPPV, and 9 fatal cases in whom IPPV had been started before biochemical investigations were carried out. Of the remaining 332 babies, 225 had estimations of capillary blood $p \mathrm{H}, \mathrm{PcO}_{2}$, and standard bicarbonate, using the Astrup micromethods, started before the age of 12 hours, and these form the basis of our present study. Of these 225 cases, 132 were born in Exeter City Hospital, and 93 were admitted from outside before the age of 12 hours. In those cases that were treated with IPPV the results of biochemical investigations carried out after starting IPPV have been excluded.

All infants were nursed in incubators, the temperature, humidity, and oxygen concentrations being adjusted, with the aim of maintaining rectal temperatures of $36-37^{\circ} \mathrm{C}$. $\left(97-98^{\circ} \mathrm{F}\right.$.). High oxygen concentrations were used whenever these were necessary to overcome cyanosis.

Tube feeding was begun at the age of 3-4 hours, in amounts of $65 \mathrm{ml} . / \mathrm{kg}$. daily, and gradually increased according to tolerance and appetite. Feeds were given 3-hourly, and indwelling gastric tubes were rarely used. During the earlier period feeds were of $10 \%$ dextrose with sodium bicarbonate in amounts varying from 10 to $25 \mathrm{mEq} / 100 \mathrm{ml}$., according to the capillary blood $p \mathrm{H}$, as recommended by Usher for intravenous therapy. Administration of sodium bicarbonate in the first few cases was continued until the $p \mathrm{H}$ rose to $7 \cdot 3$, but in later cases it was discontinued when the standard bicarbonate reached $18 \mathrm{mEq} / \mathrm{l}$., irrespective of the degree of respiratory acidosis. In the later part of the study infants were given one feed of $10 \%$ dextrose, followed by expressed breast milk.

Infants whose condition deteriorated seriously were intubated and given IPPV.

\section{Results}

Radiological examination of the chest was carried out in most cases and the findings formed a useful basis for classification, as they were found to be closely related to the clinical course and prognosis.

The first group comprised those with normal 
radiological appearances, and had no mortality. The second group will be referred to as having localized radiological abnormality, with appearances of localized atelectasis or aspiration pneumonia, and normal aeration of the remainder of the lung. Cases in this group also had a good prognosis, the only two deaths being in infants of less than 28 weeks' gestation. The third group will be referred to as having generalized radiological abnormality; the appearances varied from miliary mottling of both lung fields, to complete opacity with appearances of an 'air bronchogram'. This was the only group with a high mortality. In the fourth group which comprised cases of mild respiratory distress who were not examined radiologically, there was no mortality. The number of cases and the mortality in each group are shown in the Table.

\section{TABLE}

Number of Cases and Mortality in Infants with Respiratory Distress, Grouped according to Radiological Appearances

\begin{tabular}{|c|c|c|c|}
\hline $\begin{array}{c}\text { Appearance of Chest } \\
\qquad X \text {-ray }\end{array}$ & $\begin{array}{l}\text { No. of } \\
\text { Cases }\end{array}$ & $\begin{array}{l}\text { No. of } \\
\text { Deaths }\end{array}$ & $\begin{array}{l}\text { Mortality } \\
(\%)\end{array}$ \\
\hline $\begin{array}{l}\text { Normal } \\
\text { Localized abnormality } \\
\text { Generalized abnormality.. } \\
\text { Not examined radio- } \\
\text { logically.. } \quad . .\end{array}$ & $\begin{array}{r}48 \\
39 \\
111 \\
\\
27\end{array}$ & $\begin{array}{l}0 \\
2(2) \\
54(7) \\
0\end{array}$ & $\begin{array}{r}0 \\
5 \\
49 \\
\\
0\end{array}$ \\
\hline Total & 225 & $56(9)$ & 25 \\
\hline
\end{tabular}

* Figures in parentheses indicate the number of fatal cases of less than 28 weeks' gestation.

Incidence and degree of initial metabolic acidosis. In most cases the initial standard bicarbonate estimation was carried out before the age of 4 hours and in all cases before 12 hours. The incidence and degree of initial metabolic acidosis are shown in Fig. 1.

The incidence of severe metabolic acidosis was higher in those cases with generalized radiological abnormality, but even in this group $42 \%$ of the cases had initial standard bicarbonate levels above $18 \mathrm{mEq} / \mathrm{l}$., and of the total number of 225 babies, $53 \%$ had initial standard bicarbonate levels above $18 \mathrm{mEq} / \mathrm{l}$.

Duration of metabolic acidosis in cases not treated with sodium bicarbonate. Fig. 2 shows the percentage of patients with initial standard bicarbonate levels below $18 \mathrm{mEq} / \mathrm{l}$., whose standard bicarbonate had risen to $18 \mathrm{mEq} / \mathrm{l}$. or more at ages up to 60 hours without alkali treatment.

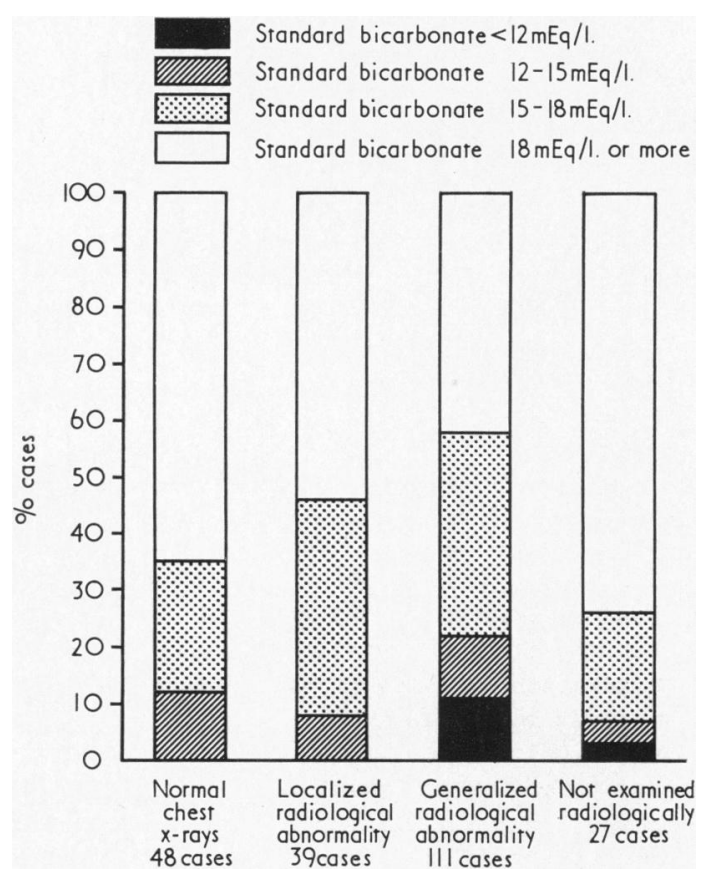

FIG. 1.-Initial standard bicarbonate levels in different types of respiratory distress.

It will be seen that infants with normal chest $x$-rays, and most of those with localized abnormality, recovered rapidly from their initial metabolic

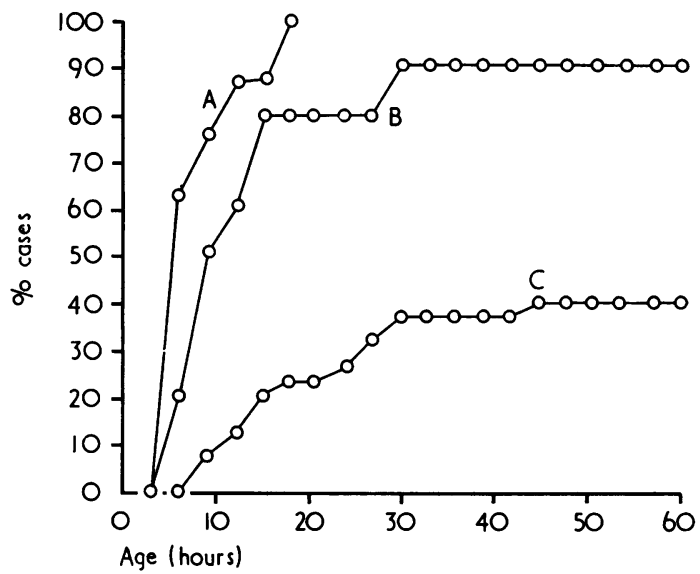

FIG. 2.-Percentage of patients with initial standard bicarbonate levels below $18 \mathrm{mEq} / \mathrm{l}$., whose standard bicarbonate reached $18 \mathrm{mEq} / \mathrm{l}$. or more at different ages. Cases not treated with alkalis, and grouped according to radiographic appearances. $A$, chest x-ray normal (8 cases); $B$, localized radiological abnormalities (10 cases); $C$, generalized radiological abnormalities (30 cases). 
acidosis, whereas in those with generalized radiological abnormality recovery took much longer.

\section{Effects of Intragastric Sodium Bicarbonate}

(A) Cases with generalized radiological abnormality. Any beneficial effect of intragastric sodium bicarbonate should be demonstrable in the group with generalized radiological abnormality, as this is the only group with prolonged metabolic acidosis and a high mortality rate. Of the 111 babies in this group, 7 were treated with THAM, 3 were only given intragastric sodium bicarbonate at a late stage, and 1 died within 3 hours of the initial estimation of standard bicarbonate, before treatment could have had any effect. After exclusion of these 11 babies, 100 remain, of whom 31 were treated with intragastric sodium bicarbonate, with 18 deaths; and 69 without alkalis, with 27 deaths.

(1) Effect of intragastric sodium bicarbonate on standard bicarbonate levels. Of the patients with generalized radiological abnormality, 56 had initial standard bicarbonate levels below $18 \mathrm{mEq} / \mathrm{l}$. Of these, 26 were treated with intragastric sodium bicarbonate and 30 without. The percentages of patients in each group whose standard bicarbonate had risen to $18 \mathrm{mEq} / 1$. or more at ages up to 60 hours are shown in Fig. 3.

It will be seen that the standard bicarbonate levels rose more quickly and in a higher percentage of cases in those given intragastric sodium bicarbonate than in those given no alkali treatment.

(2) Effect of intragastric sodium bicarbonate on $\mathrm{PCO}_{2}$ levels. In the same 56 babies with generalized radiological abnormality, the percentages in the groups treated with and without intragastric sodium bicarbonate whose $\mathrm{PCO}_{2}$ levels had fallen below $70 \mathrm{~mm} . \mathrm{Hg}$ and did not rise again are shown in Fig. 4. (One baby in whom estimations were discontinued after 28 hours, before the $\mathrm{PCO}_{2}$ had fallen below $70 \mathrm{~mm}$., has been omitted.)

It will be seen that the percentage of cases with prolonged severe respiratory acidosis was higher in those treated with intragastric sodium bicarbonate.

In order to eliminate any possible errors due to differences in initial $\mathrm{PCO}_{2}$ or differences in the numbers of fatal cases in the two groups, a more detailed study of the behaviour of the $\mathrm{PcO}_{2}$ levels in two groups of suvivors with generalized radiological abnormality was carried out. Only surviving babies with a large number of readings were used. There were 7 such babies who were given intragastric sodium bicarbonate and on the average they had a fairly high initial $\mathrm{PCO}_{2}$. Therefore, 7

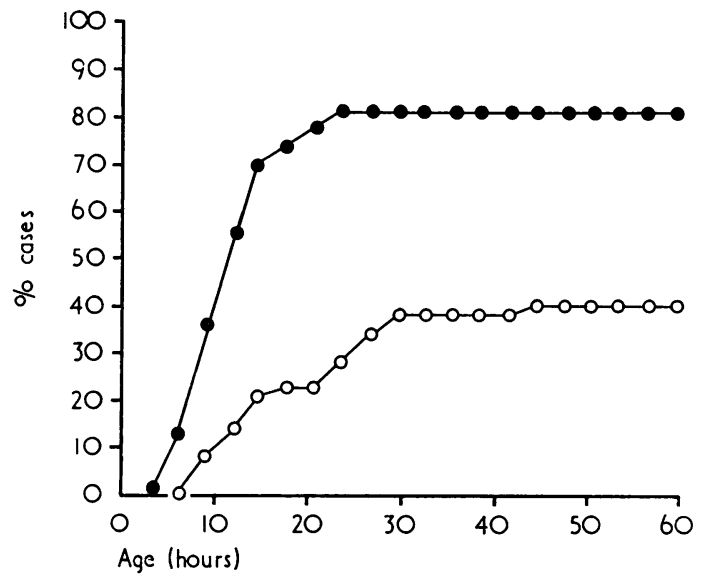

FIG. 3.-Percentage of patients with generalized radiological abnormality and initial standard bicarbonate below $18 \mathrm{mEq} / \mathrm{l}$., whose stanaiard bicarbonate had risen to 18 $m E q / l$. at different ages. 0,26 cases receiving intragastric sodium bicarbonate; $\mathrm{O}, 30$ cases not given sodium bicarbonate.

untreated babies who were found to have a similarly high average initial $\mathrm{PCO}_{2}$ level, were selected. The readings in every four-hour period of 48 hours were averaged in each group and the arithmetical regressions calculated (Simpson, Roe, and Lewontin, 1960). Results are shown in Fig. 5.

The resulting lines show a tendency for untreated babies to have a falling $\mathrm{PCO}_{2}$ in the first 48 hours

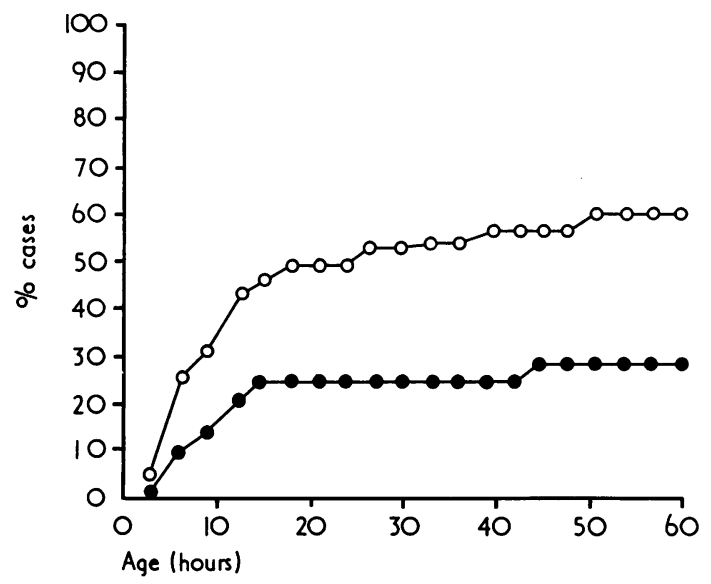

FIG. 4.-Percentage of patients with generalized radio. logical abnormality and initial standard bicarbonate below $18 \mathrm{mEq} / \mathrm{l}$., whose $\mathrm{PCO}_{2}$ had fallen below $70 \mathrm{~mm}$. at different ages. 26 cases receiving intragastric sodium bicarbonate; 0,29 cases not given sodium bicarbonate. 


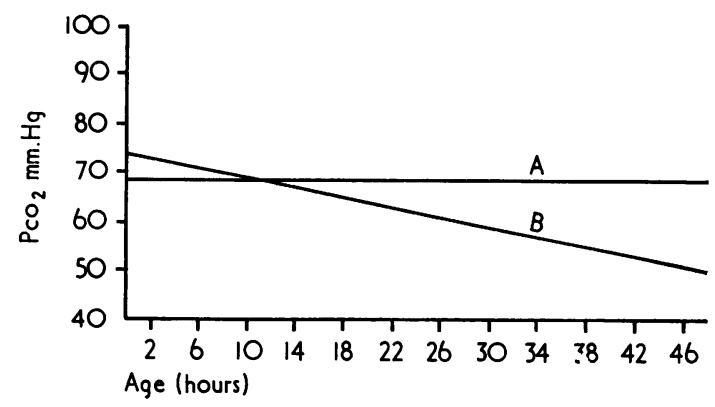

FIg. 5.-Change in $\mathrm{PCO}_{2}$ levels as a function of time (arithmetic regression) in 14 cases of respiratory distress with generalized radiological abnormality, treated with and without intragastric sodium bicarbonate. $A, 7$ cases receiving intragastric sodium bicarbonate; $B, 7$ cases not receiving sodium bicarbonate.

of life while those babies treated with intragastric sodium bicarbonate tended to retain their respiratory acidosis.

(3) Relation between total dosage of intragastric sodium bicarbonate and highest $\mathrm{PCO}_{2}$ levels. The relation between total dosage of intragastric sodium bicarbonate and the highest subsequent $\mathrm{PCO}_{2}$ levels reached is shown in Fig. 6. The babies were divided into 4 groups according to total dosage of intragastric sodium bicarbonate given per kg. body weight. In each group the average was taken of

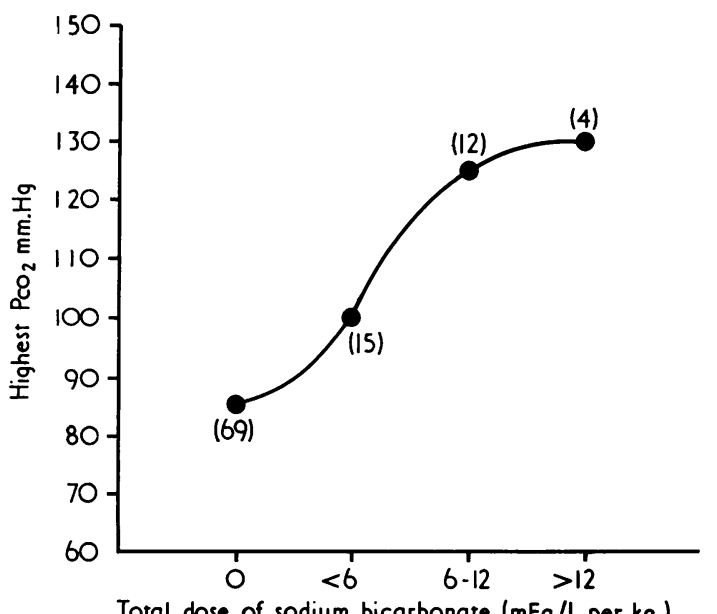

Fig. 6.-Highest $\mathrm{PCO}_{2}$ values reached, in relation to total dosage of intragastric sodium bicarbonate, in cases with generalized radiological abnormality. The figures in brackets are the numbers of cases from which a mean value was calculated. the highest $\mathrm{PcO}_{2}$ levels reached after the administration of intragastric sodium bicarbonate. (Babies on whom estimations of $\mathrm{PCO}_{2}$ were not continued for at least 6 hours after the start of feeding, with or without sodium bicarbonate, have been excluded.)

It will be seen that the highest $\mathrm{PCO}_{2}$ levels were found in those babies who had the highest total dosage of sodium bicarbonate.

(4) Effect of intragastric sodium bicarbonate on $p H$. In the same 56 babies with generalized radiological abnormality, the percentages of infants treated with and without intragastric sodium bicarbonate, whose $p \mathrm{H}$ had risen to $7 \cdot 2$ at ages up to 60 hours, are shown in Fig. 7.

It will be seen that there is little difference between the two groups, as the effect of sodium bicarbonate in correcting the metabolic acidosis is counteracted by the prolongation of respiratory acidosis.

(5) Effect of intragastric sodium bicarbonate on mortality. Mortality was $58 \%$ in the cases given intragastric sodium bicarbonate and $39 \%$ in cases given no alkali treatment. Babies born outside had a slightly higher mortality than babies born in Exeter City Hospital but were equally distributed between the two treatment groups, accounting for $42 \%$ of those given intragastric sodium bicarbonate, and $43 \%$ of those not given alkalis. Mortality was higher in babies with severe initial metabolic acidosis and also in those with lower birthweights. They were, therefore, divided into two weight groups, those below and those above 1500 g., and each was then divided into those with initial standard bicarbonate levels below and above 15 $\mathrm{mEq} / \mathrm{l}$. This gave 4 groups, in each of which the mortality in cases treated with and without intragastric sodium bicarbonate could be compared. The results are shown in Fig. 8.

It will be seen that, whatever the initial standard bicarbonate level, treatment with intragastric sodium bicarbonate did not reduce the mortality in infants of $1500 \mathrm{~g}$. and less. In the higher weight group, mortality in infants with severe metabolic acidosis was lower in those treated with intragastric sodium bicarbonate, but the difference is barely significant $\left(\chi^{2}=2 \cdot 042,0 \cdot 1<\mathrm{p}<0 \cdot 2\right)$. In those with initial standard bicarbonate levels above $15 \mathrm{mEq} / \mathrm{l}$., mortality was not reduced by intragastric sodium bicarbonate.

(B) Cases with normal chest $x$-rays or with localized radiological abnormality. Recovery from initial metabolic acidosis in both these groups of infants was so rapid that intragastric administra- 


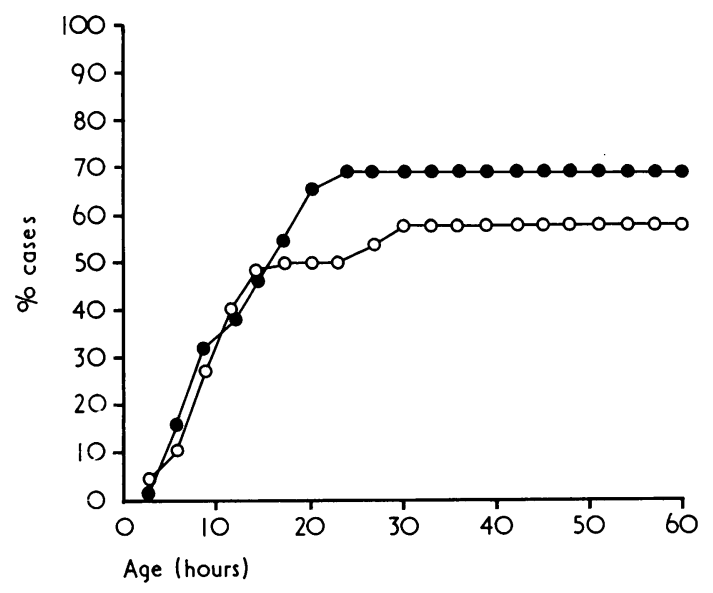

FIG. 7.-Percentage of cases with generalized radiological abnormality and initial standard bicarbonate below 18 $m E q / l$., whose $\mathrm{pH}$ had risen to $7 \cdot 2$ at different ages. 26 cases receiving intragastric sodium bicarbonate; $\bigcirc, 30$ cases not given sodium bicarbonate.

tion of sodium bicarbonate had no effect on the time taken for standard bicarbonate levels to reach $18 \mathrm{mEq} / \mathrm{l}$. or more. Recovery from respiratory acidosis was also much more rapid in these infants than in those with generalized radiological abnormality, but was slightly delayed in those given intragastric sodium bicarbonate as compared with those not given alkali treatment. Mortality was nil in infants over 28 weeks' gestation in both these groups, irrespective of the method of treatment.

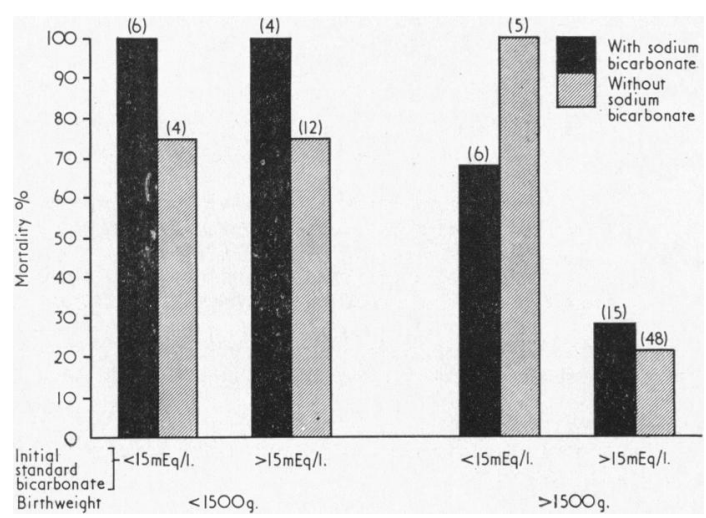

FIG. 8.-Mortality in cases with generalized radiological abnormality according to birthweight and initial standard bicarbonate levels. The number of cases is shown in brackets.

\section{Later Metabolic Acidosis}

Many infants whose standard bicarbonate levels had reached $18 \mathrm{mEq} / \mathrm{l}$. had a later fall in standard bicarbonate below this level. In the case of infants who survived, the standard bicarbonate never fell below $15 \mathrm{mEq} / 1$., whereas in fatal cases a sudden fall to levels below $15 \mathrm{mEq} / \mathrm{l}$. was a frequent occurrence, usually following apnoeic attacks.

\section{Discussion}

In the treatment of respiratory distress in the newborn correction of acidosis by administering sodium bicarbonate intravenously has become common practice, and a reduction in mortality has been reported (Usher, 1963; Hutchison et al., 1964). A recent comparison of intragastric vs. intravenous routes of glucose/bicarbonate administration showed a comparable rise in $p \mathrm{H}$ in the two groups, with a lower mortality in cases treated by the intragastric route (Abraham and Brown, 1967).

In most reported series, whatever the route of administration of sodium bicarbonate, treatment has been continued until $p \mathrm{H}$ has reached normal levels, entailing the induction of a metabolic alkalosis in order to compensate for the respiratory acidosis. The correctness of this principle has yet to be proven.

In the present study the incidence and degree of metabolic acidosis in a series of infants with respiratory distress have been investigated, and the effects of treatment with or without intragastric sodium bicarbonate have been compared. Standard bicarbonate and $\mathrm{PCO}_{2}$ rather than $p \mathrm{H}$ levels have been compared in the two groups of cases in order to distinguish between the metabolic and respiratory components of the acidosis.

In infants of 28 weeks' gestation or more, whose chest $x$-rays were normal or showed only localized abnormality, recovery from metabolic acidosis was rapid and mortality was nil. Treatment with alkalis is therefore unnecessary in such cases.

Infants whose chest $x$-rays showed generalized abnormality had a higher incidence of severe metabolic acidosis initially, but when cyanosis could be relieved the metabolic acidosis slowly corrected itself. A more rapid recovery from metabolic acidosis in many of these infants could be achieved by intragastric administration of sodium bicarbonate, but this treatment delayed recovery from respiratory acidosis.

Persisting metabolic acidosis in cases of severe respiratory distress is almost always an indication of continuing hypoxia. Unless oxygen tensions are being monitored frequently, the raising of standard 
bicarbonate levels by administering sodium bicarbonate may prevent the recognition of continuing metabolic acidosis as a valuable indication that oxygen concentrations need to be increased.

It might be expected that any beneficial effect of treatment with intragastric sodium bicarbonate on mortality would be less noticeable in cases where the initial metabolic acidosis was mild. This has been confirmed in the present study, which has shown no reduction in mortality following intragastric administration of sodium bicarbonate in cases with generalized radiological abnormality and initial standard bicarbonate levels of $15 \mathrm{mEq} / \mathrm{l}$. or more. Treatment with intragastric sodium bicarbonate is not therefore indicated in this group of cases.

Mortality was high in those cases with generalized radiological abnormality and severe initial metabolic acidosis, whether intragastric sodium bicarbonate was given or not. Correction of the metabolic acidosis by intragastric administration of sodium bicarbonate did not reduce the mortality in infants weighing $1500 \mathrm{~g}$. and less. Although the mortality was lower in infants over $1500 \mathrm{~g}$. who were given intragastric sodium bicarbonate, the difference was not significant.

Since severe metabolic acidosis in cases of respiratory distress is indicative of hypoxia and is usually accompanied by respiratory acidosis, correction of metabolic acidosis alone, without efforts to relieve hypoxia and to improve ventilation would not be expected to reduce mortality greatly. Treatment by means of IPPV is the rational approach in such cases, when $100 \% \mathrm{O}_{2}$ has failed to relieve hypoxia and metabolic acidosis, and particularly when respiratory acidosis is also severe. Our experience with IPPV so far confirms that of Reid and Tunstall (1966) that in most cases IPPV corrects both respiratory and metabolic acidosis, and leads us to conclude that many of the infants treated during the earlier part of this study should have been given IPPV earlier in the course of their illness. In a very small minority of cases metabolic acidosis is not completely relieved by IPPV, and in these cases alone there may be an indication for treatment with intragastric sodium bicarbonate.

\section{Summary}

The incidence of metabolic acidosis has been investigated in 225 infants with respiratory distress. In infants who initially had standard bicarbonate levels below $18 \mathrm{mEq} / \mathrm{l}$. (less than half the total) spontaneous recovery from metabolic acidosis was rapid in those whose chest radiographs were normal or showed only localized abnormality, but was delayed in many with generalized radiological abnormality.

Administration of intragastric sodium bicarbonate to a group of infants with generalized radiological abnormality speeded recovery from metabolic acidosis, but was often followed by a more severe and prolonged respiratory acidosis. Mortality was not reduced.

The value of treatment with intragastric sodium bicarbonate in neonatal respiratory distress is strictly limited. It may be of some value in the small minority of cases in which metabolic acidosis persists despite treatment by means of intermittent positive pressure respiration.

We wish to thank Dr. F. S. W. Brimblecombe and Dr. L. Haas for allowing us to study patients admitted under their care and for much helpful advice; Dr. J. Sheach for radiological opinions; and Sister J. Boxall and the staff of the Special Care Baby Unit for their devoted care of the patients.

This work was made possible by a grant from the South Western Regional Hospital Board, and a Research Fellowship from Vickers Ltd.

\section{REFERENCES}

Abraham, J. M., and Brown, R. J. K. (1967). Comparison of intragastric and intravenous routes of glucose/bicarbonate administration in respiratory distress syndrome. Brit. med f., 3, 640 .

Gairdner, D. (1965). Respiratory distress in the newborn. In Recent Advances in Paediatrics, 3rd ed., p. 54. Ed. by D.Gairdner. Churchill, London.

Hutchison, J. H., Kerr, M. M., Douglas, T. A., Inall, J. A., and Crosbie, J. C. (1964). A therapeutic approach in 100 cases of the respiratory distress syndrome of the newborn infant. Pediatrics, 33, 956.

- - McPhail, M. F. M., Douglas, T. A., Smith, G., Norman, J. N., and Bates, E. H. (1962). Studies in the treatment of the pulmonary syndrome of the newborn. Lancet, 2, 465.

Reid, D. H. S., and Tunstall, M. E. (1966). The respiratory distress syndrome of the newborn. Anaesthesia, 21, 72 .

Simpson, G. G., Roe, A., and Lewontin, R. C. (1960). Quantitative Zoology. Harcourt, Brace, New York.

Usher, R. (1959). The respiratory distress syndrome of prematurity. I. Changes in potassium in the serum and the electrocardiogram and effects of therapy. Pediatrics, 24, 562.

- (1960). Management of metabolic changes in the respiratory distress syndrome of prematurity. Amer. F. Dis. Child., 100, 485.

(1961). The respiratory distress syndrome of prematurity. Clinical and therapeutic aspects. Pediat. Clin. N. Amer., 8, 525.

- (1963). Reduction of mortality from respiratory distress syndrome of prematurity with early administration of intravenous glucose and sodium bicarbonate. Pediatrics, 32, 966.

Warley, M. A., and Gairdner, D. (1962). Respiratory distress syndrome of the newborn-principles in treatment. Arch. Dis. Childh., 37, 455. 